\title{
The Effect of Lead Time and Demand Uncertainties in $(r, q)$ Inventory Systems
}

\author{
Jing-Sheng Song \\ The Fuqua School of Business, Duke University, Durham, North Carolina 27708, \\ and Antai College of Economics and Management, Shanghai Jiao Tong University, \\ Shanghai 200052, China, jssong@duke.edu \\ Hanqin Zhang \\ Institute of Applied Mathematics, Academia Sinica, Beijing 100080, China, \\ and Business School, National University of Singapore, Singapore, hanqin@amt.ac.cn \\ Yumei Hou \\ Yanshan University, Qinhuangdao 066004, Hebei Province, China, hym_1220@163.com \\ Mingzheng Wang \\ Dalian University of Technology, Dalian 116024, China, mzhwang@dlut.edu.cn
}

\begin{abstract}
We study a single-item $(r, q)$ inventory system, where $r$ is the reorder point and $q$ is the order quantity. The demand is a compound-Poisson process. We investigate the behavior of the optimal policy parameters and the long-run average cost of the system in response to stochastically shorter or less-variable lead times. We show that although some of the properties of the base-stock system can be extended to this more general model, some cannot. The same findings also apply when the comparison is conducted on the lead-time demand distributions.
\end{abstract}

Subject classifications: inventory system; reorder-point/order quantity; stochastic lead time; variability; optimal policy; stochastic comparison.

Area of review: Manufacturing, Service, and Supply Chain Operations.

History: Received January 2007; revisions received August 2007, May 2008, December 2008; accepted January 2009.

Published online in Articles in Advance October 7, 2009.

\section{Introduction}

We consider a single-item inventory system driven by a pure Poisson demand process and governed by an $(r, q)$ replenishment policy. (We discuss extensions to compoundPoisson demand in §5.) Under such a policy, an order of fixed size $q$ is placed when the inventory position drops to level $r$. Order lead times are stochastic and generated by an exogenous, sequential supply process, as discussed in Zipkin (1986, 2000). This lead-time model ensures that orders never cross. Under standard cost assumptions (fixed plus linear order costs and linear or convex inventory/backorder costs), an $(r, q)$ policy is indeed optimal; see Veinott (1965) and Zipkin (2000). The objective of this paper is to demonstrate the impact of the lead-time distribution on the optimal reorder point $r^{*}$, the optimal order size $q^{*}$, and order-up-to level $r^{*}+q^{*}$, as well as the optimal average cost. More precisely, we specify how these policy parameters and performance measures vary as the lead-time distribution becomes stochastically smaller or less variable. The main results are summarized as follows:

(i) For a fixed order quantity $q$, the optimal reorder point $r^{*}(q)$ is lower if the lead time (or the lead-time demand) is stochastically smaller. On the other hand, when the lead time or the lead-time demand is less variable (while the mean stays the same), $r^{*}(q)$ is lower, if and only if a holding-backorder cost ratio is above a threshold. In addition, this latter variability effect decreases in $q$.

(ii) When $q$ is also optimized, a stochastically smaller lead time or lead-time demand results in lower $r^{*}$ and $r^{*}+q^{*}$

(iii) In general, $r^{*}$ can be either lower or higher in response to a less-variable lead-time demand. However, a lower $r^{*}$ is always accompanied by a lower $r^{*}+q^{*}$. On the other hand, a higher $r^{*}$ is accompanied by a higher $r^{*}+q^{*}$ only under certain conditions.

(iv) Assuming the lead-time demands can be approximated by normal distributions, a less variable lead-time demand results in (a) a smaller $q^{*}$ if the fixed ordering cost is above a threshold; and (b) a lower order-up-to level $r^{*}+q^{*}$ if the unit backorder cost is higher than the unit holding cost rate.

(v) A stochastically smaller lead time or lead-time demand may not necessarily lead to a lower optimal system cost, whereas a less variable lead time or lead-time demand always reduces the system cost.

(vi) Finally, the above results hold for compoundPoisson demand processes. They also hold for renewal 
and continuous demand processes, provided the stochastic comparison is on the basis of the lead-time demand distributions.

Song (1994a) studies a special case of the system considered here-there is no fixed order cost, and therefore a base-stock policy (corresponding to $q=1$ ) is optimal. Our results (i) and (v) above are extensions of hers. (Her findings are also supported by Chopra et al. 2004.) Zheng (1992) shows that the optimal order quantity of any stochastic $(r, q)$ system is no less than that of the system with deterministic demand. This is a special case of our investigation of the effect of lead-time demand variability. As shown in \$4, Zheng's result cannot be extended to the general case (i.e, when both systems have uncertain demand and lead time). Assuming that lead-time demand follows a normal distribution $N\left(\mu, \sigma^{2}\right)$, Zipkin (2000) illustrates numerically that the optimal order quantity $q^{*}$ increases in $\sigma$. Our result (iv)(a) establishes this property analytically, provided the fixed ordering cost is not too small.

There has been growing interest in the inventory literature to apply stochastic orders to study the effect of lead time and/or demand uncertainties. Examples include Gerchak and Mossman (1992), Song (1994a, b), Ridder et al. (1998), Song and Yao (2002), Lu et al. (2003), Iyer and Jain (2003), Gupta and Cooper (2005), and Jemaï and Karaesman (2005). However, all these studies concentrate on the base-stock or newsvendor systems. Our paper appears to be the first attempt to conduct stochastic comparison of $(r, q)$ systems.

We are aware of only two previous studies on the effect of lead-time variabilities in $(r, q)$ systems. Bagchi et al. (1986) investigate the impact of lead-time variabilities on stockouts and stockout risk in an $(r, q)$ system with i.i.d. demand. Song and Zipkin (1996) discuss the joint effect of lead-time variability and lot size $q$ on net inventory. They assume i.i.d. lead times. This implies a parallel processing environment, which is different from our sequential supply system. Whereas these papers focus on the behavior of key performance measures, we are interested in the behavior of the optimal policy and cost.

The rest of this paper is organized as follows. Sections 2-4 focus on the basic model with a Poisson demand process. Section 2 introduces the notation and presents some preliminaries. Section 3 studies the effect of a stochastically shorter lead time, whereas $\S 4$ investigates the impact of a less-variable lead time. In $\$ 5$, we discuss several extensions, including compound-Poisson, renewal, and continuous demand processes. Some long proofs are provided in the appendix.

\section{Preliminaries}

We compare two single-item continuous-review $(r, q)$ inventory systems, indexed by subscript $i, i=1,2$. The features of both systems are identical except for the lead-time distributions. In particular, the demand of each system is a Poisson process with rate $\lambda$. There is a linear inventoryholding cost at a unit rate $h$. Unsatisfied demand is backlogged, incurring a unit backorder penalty cost rate $p$. For each replenishment order, there is a fixed ordering cost $K$. The following parameters depend on the system:

$L_{i}=$ lead time of system $i$, a continuous random variable;

$D_{i}=$ lead time demand in system $i$, a discrete random variable;

$\Psi_{i}=$ cumulative distribution function of $D_{i}$;

$\psi_{i}=$ probability mass function of $D_{i}$; and

$U_{i}(q)=$ a uniform $\{1, \ldots, q\}$ random variable independent of $D_{i}$.

Let $\Delta$ be the difference operator, i.e., for any discrete function $f(x)$,

$\Delta f(x)=f(x+1)-f(x)$.

When a function has several discrete variables, we use $\Delta_{v}$ to denote the difference with respect to variable $v$.

Denote by $G_{i}(y)$ the average cost of a base-stock policy with a base-stock level $y$ in system $i$. Then it is well known that

$$
\begin{aligned}
G_{i}(y) & =h \cdot \mathrm{E}\left[\left(y-D_{i}\right)^{+}\right]+p \cdot \mathrm{E}\left[\left(D_{i}-y\right)^{+}\right] \\
& =(h+p) \sum_{u=0}^{y-1} \Psi_{i}(u)+p \lambda \cdot \mathrm{E} L_{i}-p y .
\end{aligned}
$$

$G_{i}(y)$ is convex in $y$ and $\Delta G_{i}(y)=(p+h) \Psi_{i}(y)-p$. The optimal base-stock level $y_{i}^{*}$ satisfies

$y_{i}^{*}=\min \left\{y \mid \Delta G_{i}(y) \geqslant 0\right\}=\min \left\{y \mid \Psi_{i}(y) \geqslant \frac{p}{p+h}\right\}$.

The expected long-run average cost of system $i$ under an $(r, q)$ policy can be expressed as the simple average of $q$ base-stock system costs:

$c_{i}(r, q)=\frac{\lambda K+\sum_{y=r+1}^{r+q} G_{i}(y)}{q}=\frac{1}{q} \sum_{x=1}^{q} G_{i}(r+x)+\frac{\lambda K}{q}$.

Note that when $q=1$ the policy reduces to a base-stock policy with base-stock level $r+1$.

For any fixed $q, c_{i}(r, q)$ is convex in $r$. Let $r_{i}^{*}(q)$ be its smallest minimizer, i.e.,

$c_{i}^{*}(q)=\min _{r} c_{i}(r, q)$ and $r_{i}^{*}(q)=\min \left\{r \mid c_{i}(r, q)=c_{i}^{*}(q)\right\}$.

Then, $r_{i}^{*}(q)$ is the optimal reorder point for the given $q$. The optimal order quantity is thus $q_{i}^{*}=\arg \min _{q} c_{i}^{*}(q)$. When there are multiple minimizers, we choose the smallest one. The optimal reorder point is then $r_{i}^{*}=r_{i}^{*}\left(q^{*}\right)$.

Our primary concerns in this paper are the following: Suppose that $L_{1}$ is "longer" than $L_{2}$ in the stochastic sense. 
Does this imply $r_{1}^{*}(q) \geqslant r_{2}^{*}(q)$ for any fixed $q$ ? How should $r_{i}^{*}, q_{i}^{*}$, and $c_{i}\left(r_{i}^{*}, q_{i}^{*}\right)$ be ordered? Will these orders change if $L_{i}$ have the same mean but $L_{1}$ is "more variable"? Sections 3 and 4 address these questions separately.

To facilitate the analysis throughout the paper, in the remainder of this section we review some existing results and present some preliminary findings.

We first present a result that relates $r_{i}^{*}(q)$ and $r_{i}^{*}(q)+q$ to base-stock systems. It is the discrete analogue of a property in Gallego (1998) for continuous demand $(r, q)$ systems. Consider system $i$. For any fixed $q$, define

$C_{i}(q, y)=\sum_{x=1}^{q} \operatorname{Pr}\left\{D_{i} \leqslant y+x\right\}=\sum_{x=1}^{q} \Psi_{i}(y+x)$.

Note that $r_{i}^{*}(q)$ is the smallest $r$ that satisfies

$\Delta_{r} c_{i}(r, q)=\frac{1}{q} \sum_{x=1}^{q} \Delta G_{i}(r+x)=(p+h) \frac{C_{i}(q, r)}{q}-p \geqslant 0$.

We have:

Lemma 1. For any fixed $q$,

$$
\begin{aligned}
r_{i}^{*}(q) & =\min \left\{y \mid \frac{1}{q} C_{i}(q, y) \geqslant \frac{p}{p+h}\right\} \\
& =\min \left\{y \mid \operatorname{Pr}\left\{D_{i}-U_{i}(q) \leqslant y\right\} \geqslant \frac{p}{p+h}\right\}, \\
r_{i}^{*}(q) & +q=\min \left\{y \mid \operatorname{Pr}\left\{D_{i}+U_{i}(q) \leqslant y\right\} \geqslant \frac{p}{p+h}\right\} .
\end{aligned}
$$

That is, $r_{i}^{*}(q)$ and $r_{i}^{*}(q)+q$ are the optimal base-stock levels for systems with holding cost rate $h$, penalty cost rate $p$, and lead-time demands $D_{i}-U_{i}(q)$ and $D_{i}+U_{i}(q)$, respectively.

Note that, for any fixed $q$, the study of the ordering of $r_{i}^{*}(q)$ is equivalent to that of $r_{i}^{*}(q)+q$. With Lemma 1 , the latter reduces to that of $D_{i}+U_{i}(q)$ and the corresponding result for the base-stock system.

In addition, these functions have the following properties; the proofs are given in the appendix.

Lemma 2. For any fixed $i$ and $y$,

(i) $C_{i}(q, y) / q$ is nondecreasing in $q$; and

(ii) $r_{i}^{*}(q)$ is nonincreasing and $r_{i}^{*}(q)+q$ is nondecreasing.

(Property (ii) is the discrete analogue of Part 3 of Lemma 3 in Zheng 1992.)

We now review the algorithm by Federgruen and Zheng (1992) for computing $\left(r_{i}^{*}, q_{i}^{*}\right)$. First, these authors show that for any fixed $q$, the $q$ smallest $G_{i}(\cdot)$ values are achieved at contiguous points and $c_{i}^{*}(q)$ is achieved if the sum in (2) consists of these values. Based on this observation, the optimal reorder points $r_{i}^{*}(1), \ldots, r_{i}^{*}(q)$ are easy to identify. In particular, these $q$ consecutive points can be generated inductively. Let $y_{i}^{1}=y_{i}^{*}$ be the smallest integer that minimizes $G_{i}(\cdot)$ over all integers. Assuming that $\left\{y_{i}^{1}, \ldots, y_{i}^{k}\right\}$ have been generated for some $k \geqslant 1$, define $\mathscr{L}_{i}(k)=\min \left\{y_{i}^{1}, \ldots, y_{i}^{k}\right\}$ and $\mathscr{R}_{i}(k)=\max \left\{y_{i}^{1}, \ldots, y_{i}^{k}\right\}$. Let

$y_{i}^{k+1}= \begin{cases}\mathscr{L}_{i}(k)-1, & \text { if } G_{i}\left(\mathscr{L}_{i}(k)-1\right) \leqslant G_{i}\left(\mathscr{R}_{i}(k)+1\right), \\ \mathscr{R}_{i}(k)+1, & \text { otherwise. }\end{cases}$

Then, due to the unimodality of $-G_{i}(\cdot),\left\{y_{i}^{1}, \ldots, y_{i}^{q}\right\}$ are contiguous and $G_{i}\left(y_{i}^{k}\right)$ is the $k$ th smallest $G_{i}$ value, $k=1, \ldots, q$. From this construction, for any given integer $q \geqslant 1$,

$r_{i}^{*}(q)=\mathscr{L}_{i}(q)-1, \quad r_{i}^{*}(q)+q=\mathscr{R}_{i}(q)$.

Moreover, suppose that $y_{i}^{m}=r_{i}^{*}(q)$ for some $m$. Then, $m>q$ and $y_{i}^{m} \leqslant y_{i}^{k}, k=1, \ldots, m$. Also,

$c_{i}^{*}(q)=\frac{\lambda K+\sum_{j=1}^{q} G_{i}\left(y_{i}^{j}\right)}{q}$.

Second, Federgruen and Zheng (1992) show that the sequence $\left\{y_{i}^{k}\right\}$ can be used to determine the optimal order quantity $q^{*}$ :

Lemma 3 (Federgruen And Zheng 1992). $q_{i}^{*}$ is the smallest integer $q$ such that

$c_{i}^{*}(q) \leqslant G_{i}\left(y_{i}^{q+1}\right)$.

From their proof, we can also see that

$q<q_{i}^{*} \quad$ if and only if $\quad c_{i}^{*}(q)>G_{i}\left(y_{i}^{q+1}\right)$.

\section{The Effect of a Shorter Lead Time}

In this section, we study the effects of the magnitude of the lead time. We use the usual stochastic order $\geqslant_{\text {st }}$ (see Shaked and Shanthikumar 1994) to measure the magnitudes of two random variables.

The following results are drawn from Song (1994a) on base-stock systems.

Lemma 4 (Song 1994a). If $L_{1} \geqslant_{\mathrm{st}} L_{2}$, then

(i) $D_{1} \geqslant_{\mathrm{st}} D_{2}$;

(ii) $\Delta G_{1}(y) \leqslant \Delta G_{2}(y)$ for all $y$; and

(iii) $y_{1}^{*} \geqslant y_{2}^{*}$, where $y_{i}^{*}(i=1,2)$ are defined by (1). Thus, a stochastically shorter lead time requires a lower optimal base-stock level.

For any given $q$, because independent sums preserve the usual stochastic order, Lemma 4 part (i) implies $D_{1}+$ $U_{1}(q) \geqslant_{\text {st }} D_{2}+U_{2}(q)$. Now, replacing $D_{i}$ by $D_{i}+U_{i}(q)$ in Lemma 4 and applying Lemma 1, we obtain:

THEOREM 1. If $L_{1} \geqslant_{\mathrm{st}} L_{2}$, then $r_{1}^{*}(q) \geqslant r_{2}^{*}(q)$ for any given $q$. 
That is, with the order quantity fixed, a stochastically shorter lead time requires a lower optimal reorder point.

When the order quantity is optimized, we have:

THEOREM 2. If $L_{1} \geqslant{ }_{\mathrm{st}} L_{2}$, then $r_{1}^{*} \geqslant r_{2}^{*}$ and $r_{1}^{*}+q_{1}^{*} \geqslant$ $r_{2}^{*}+q_{2}^{*}$.

Proof. Consider four possible cases:

Case 1. $r_{1}^{*} \geqslant r_{2}^{*}$ and $r_{1}^{*}+q_{1}^{*} \geqslant r_{2}^{*}+q_{2}^{*}$;

Case 2. $r_{1}^{*} \geqslant r_{2}^{*}$ and $r_{1}^{*}+q_{1}^{*}<r_{2}^{*}+q_{2}^{*}$;

Case 3. $r_{1}^{*}<r_{2}^{*}$ and $r_{1}^{*}+q_{1}^{*} \geqslant r_{2}^{*}+q_{2}^{*}$; and

Case 4. $r_{1}^{*}<r_{2}^{*}$ and $r_{1}^{*}+q_{1}^{*}<r_{2}^{*}+q_{2}^{*}$.

To prove the theorem, we show that the last three cases are impossible. We first examine Case 2. Suppose that the inequalities in this case hold. Because $r_{2}^{*} \leqslant r_{1}^{*}<r_{1}^{*}+q_{1}^{*}+$ $1 \leqslant r_{2}^{*}+q_{2}^{*}$, there must exist $\hat{q}$ such that

$y_{2}^{\hat{q}+1}=r_{1}^{*}+q_{1}^{*}+1 \in\left\{y_{2}^{1}, \ldots, y_{2}^{q_{2}^{*}}\right\}$.

Recall that $\left\{r_{1}^{*}+1, \ldots, r_{1}^{*}+q_{1}^{*}\right\}$ achieve the $q_{1}^{*}$ smallest $G_{1}$ values. We thus have $G_{1}\left(r_{1}^{*}+q_{1}^{*}+1\right) \geqslant G_{1}\left(r_{1}^{*}+1\right)$. According to Lemma 4(ii), this implies $G_{2}\left(r_{1}^{*}+q_{1}^{*}+1\right) \geqslant$ $G_{2}\left(r_{1}^{*}+1\right)$. Hence, if the set of the contiguous smallest $G_{2}$ values contains $r_{1}^{*}+q_{1}^{*}+1$, then it must contain $r_{1}^{*}+1$. In other words, $r_{1}^{*}+1 \in\left\{y_{2}^{1}, \ldots, y_{2}^{\hat{q}+1}\right\}$. Hence,

$\left\{y_{1}^{1}, \ldots, y_{1}^{q_{1}^{*}}\right\}=\left\{r_{1}^{*}+1, \ldots, r_{1}^{*}+q_{1}^{*}\right\} \subseteq\left\{y_{2}^{1}, \ldots, y_{2}^{\hat{q}+1}\right\}$

and

$q_{2}^{*} \geqslant \hat{q}+1>q_{1}^{*}$.

By (6), we must have $c_{2}^{*}(\hat{q})>G_{2}\left(y_{2}^{\hat{q}+1}\right)$, which leads to

$$
\begin{aligned}
& G_{2}\left(y_{2}^{\hat{q}+1}\right)-c_{2}^{*}(\hat{q}) \\
& \quad=\frac{\sum_{j=1}^{\hat{q}}\left[G_{2}\left(y_{2}^{\hat{q}+1}\right)-G_{2}\left(y_{2}^{j}\right)\right]-\lambda K}{\hat{q}}<0 .
\end{aligned}
$$

Note that from (6) we have $c_{1}^{*}\left(q_{1}^{*}\right) \leqslant G_{1}\left(y_{1}^{q_{1}^{*}+1}\right)$, whereas from (7) we have $G_{1}\left(y_{1}^{q_{1}^{*}+1}\right) \leqslant G_{1}\left(y_{2}^{\hat{q}+1}\right)$. So, $c_{1}^{*}\left(q_{1}^{*}\right) \leqslant$ $G_{1}\left(y_{2}^{\hat{q}+1}\right)$. This yields

$$
\begin{aligned}
& G_{1}\left(y_{2}^{\hat{q}+1}\right)-c_{1}^{*}\left(q_{1}^{*}\right) \\
& \quad=\frac{\sum_{j=1}^{q_{1}^{*}}\left[G_{1}\left(y_{2}^{\hat{q}+1}\right)-G_{1}\left(y_{1}^{j}\right)\right]-\lambda K}{q_{1}^{*}} \geqslant 0 .
\end{aligned}
$$

On the other hand, from (7) we know that $y_{2}^{\hat{q}+1} \geqslant y_{1}^{j}$, $j=1, \ldots, q_{1}^{*}$. Thus, by Lemma 4(ii), we have

$\left[G_{1}\left(y_{2}^{\hat{q}+1}\right)-G_{1}\left(y_{1}^{j}\right)\right] \leqslant\left[G_{2}\left(y_{2}^{\hat{q}+1}\right)-G_{2}\left(y_{1}^{j}\right)\right], \quad j=1, \ldots, q_{1}^{*}$.

This implies

$$
\begin{aligned}
& \sum_{j=1}^{q_{1}^{*}}\left[G_{1}\left(y_{2}^{\hat{q}+1}\right)-G_{1}\left(y_{1}^{j}\right)\right]-\lambda K \\
& \quad \leqslant \sum_{j=1}^{q_{1}^{*}}\left[G_{2}\left(y_{2}^{\hat{q}+1}\right)-G_{2}\left(y_{1}^{j}\right)\right]-\lambda K \\
& \quad \leqslant \sum_{j=1}^{\hat{q}}\left[G_{2}\left(y_{2}^{\hat{q}+1}\right)-G_{2}\left(y_{2}^{j}\right)\right]-\lambda K,
\end{aligned}
$$

where the last inequality holds because of (7), (8), and $G_{2}\left(y_{2}^{\hat{q}+1}\right)-G_{2}\left(y_{2}^{j}\right) \geqslant 0,1 \leqslant j \leqslant \hat{q}$. However, this contradicts (9) and (10), so Case 2 cannot hold.

Next we consider Case 3. Because $r_{1}^{*}<r_{2}^{*}<r_{2}^{*}+q_{2}^{*} \leqslant$ $r_{1}^{*}+q_{1}^{*}$, there must exist $\tilde{q}$ such that

$y_{1}^{\tilde{q}+1}=r_{2}^{*} \quad$ and $\quad \tilde{q}+1 \leqslant q_{1}^{*}$.

Similar to (7) and (8), we have

$\tilde{q} \geqslant q_{2}^{*} \quad$ and $\quad\left\{y_{2}^{1}, \ldots, y_{2}^{q_{2}^{*}}\right\} \subseteq\left\{y_{1}^{1}, \ldots, y_{1}^{\tilde{q}+1}\right\}$.

Using (3), (12), and (13), we obtain

$c_{1}^{*}(\tilde{q})>G_{1}\left(y_{1}^{\tilde{q}+1}\right)$ and $c_{2}^{*}\left(q_{2}^{*}\right) \leqslant G_{2}\left(y_{1}^{\tilde{q}+1}\right)$.

Note that from (5) and (12),

$y_{1}^{\tilde{q}+1} \leqslant y_{1}^{j}, \quad j=1, \ldots, \tilde{q}$.

Similarly to (11), using (13)-(15), we can prove that Case 3 is also impossible.

Finally, we consider Case 4. Because $\Delta G_{1}(y) \leqslant \Delta G_{2}(y)$ (see Lemma 4), we know that $r_{1}^{*}<r_{1}^{*}+q_{1}^{*}<r_{2}^{*}<r_{2}^{*}+$ $q_{2}^{*}$ is impossible. Thus, under the condition of Case 4 , we have $r_{1}^{*}<r_{2}^{*} \leqslant r_{1}^{*}+q_{1}^{*}<r_{2}^{*}+q_{2}^{*}$. Let $y_{2}^{\bar{q}}=r_{2}^{*}$. Then, $y_{2}^{\bar{q}} \notin$ $\left\{r_{2}^{*}+1, \ldots, r_{2}^{*}+q_{2}^{*}\right\}$, but $y_{2}^{q} \in\left\{r_{1}^{*}+1, \ldots, r_{1}^{*}+q_{1}^{*}\right\}$. Hence,

$G_{2}\left(y_{2}^{\bar{q}}\right)>G_{2}\left(r_{2}^{*}+q_{2}^{*}\right)$.

Furthermore, because $r_{2}^{*}+q_{2}^{*} \notin\left\{r_{1}^{*}+1, \ldots, r_{1}^{*}+q_{1}^{*}\right\}$, we also have

$G_{1}\left(y_{2}^{\bar{q}}\right) \leqslant G_{1}\left(r_{2}^{*}+q_{2}^{*}\right)$.

On the other hand, by Lemma 4(ii) and $y_{2}^{\bar{q}}<r_{2}^{*}+q_{2}^{*}$,

$G_{1}\left(r_{2}^{*}+q_{2}^{*}\right)-G_{1}\left(y_{2}^{\bar{q}}\right) \leqslant G_{2}\left(r_{2}^{*}+q_{2}^{*}\right)-G_{2}\left(y_{2}^{\bar{q}}\right)$,

which contradicts (16) and (17). Thus, Case 4 is impossible. This concludes the proof of the theorem.

Theorem 2 shows that a stochastically shorter lead time has monotonic effects on the optimal reorder point and optimal order-up-to level. The effects on the optimal average cost and optimal order quantity, however, are more intricate. A stochastically shorter lead time does not necessarily result in a lower average cost or a smaller order quantity, as illustrated by the following example.

Example 1. Consider $p=9, h=1, \lambda=5, K=32$, $\operatorname{Pr}\left\{L_{1}=1\right\}=0.9, \operatorname{Pr}\left\{L_{1}=0.1\right\}=0.1, \operatorname{Pr}\left\{L_{2}=1\right\}=0.5$, and $\operatorname{Pr}\left\{L_{2}=0.1\right\}=0.5$. Thus, $L_{1} \geqslant_{\text {st }} L_{2}$. With these data, we have $r_{1}^{*}=2, q_{1}^{*}=20, c_{1}^{*}\left(r_{1}^{*}, q_{1}^{*}\right)=18.4009$, and $r_{2}^{*}=0$, $q_{2}^{*}=21, c_{2}^{*}\left(r_{2}^{*}, q_{2}^{*}\right)=18.8750$. Thus, $L_{1} \geqslant_{\mathrm{st}} L_{2}$ does not imply $q_{1}^{*} \geqslant q_{2}^{*}$ or $c_{1}^{*} \geqslant c_{2}^{*}$.

On the other hand, if $p=10, K=20$, and $\operatorname{Pr}\left\{L_{1}=10\right\}$ $0.9, \operatorname{Pr}\left\{L_{1}=0.1\right\}=0.1$, while the other data remain the same, we obtain $r_{1}^{*}=52, q_{1}^{*}=18, c_{1}^{*}\left(r_{1}^{*}, q_{1}^{*}\right)=25.9906$, and $r_{2}^{*}=2, q_{2}^{*}=16, c_{2}^{*}\left(r_{2}^{*}, q_{2}^{*}\right)=15.8728$. Thus, again, $L_{1} \geqslant_{\text {st }} L_{2}$. However, we now have $q_{1}^{*}>q_{2}^{*}$ and $c_{1}^{*}>c_{2}^{*}$. Combining the two cases, we see that $q_{i}^{*}$ and $c_{i}^{*}$ can be ordered either way. 
Below are some bounds on the difference of the optimal average costs of the two systems; their proofs are in the appendix.

THEOREM 3. If $L_{1} \geqslant_{\mathrm{st}} L_{2}$, then for any given $(r, q)$ policy, the long-run average costs $c_{i}(r, q)(i=1,2)$ satisfy

$$
\begin{aligned}
-h \lambda\left[\mathrm{E} L_{1}-\mathrm{E} L_{2}\right] & \leqslant c_{1}(r, q)-c_{2}(r, q) \\
& \leqslant p \lambda\left[\mathrm{E} L_{1}-\mathrm{E} L_{2}\right] .
\end{aligned}
$$

Furthermore, $c_{1}\left(r_{1}^{*}, q_{1}^{*}\right)-c_{2}\left(r_{2}^{*}, q_{2}^{*}\right)$ has the same bounds.

\section{The Effect of a Less-Variable Lead Time}

In this section, we assume that $\mathrm{E}\left[L_{1}\right]=\mathrm{E}\left[L_{2}\right]$ and focus on the variability of $L_{i}$.

Perhaps the most popular notion for comparing the variability of two random variables is the convex order $\geqslant_{\mathrm{cx}}$ (see Shaked and Shanthikumar 1994). It is known that $X \geqslant_{\mathrm{cx}} Y$ implies (a) $\mathrm{E}[X]=\mathrm{E}[Y]$ and $\operatorname{Var}[X] \geqslant \operatorname{Var}[Y]$; (b) there exists a random variable $\epsilon$, with $\mathrm{E}(\epsilon)=0$ and $\mathrm{E}(\epsilon \mid X) \geqslant 0$ almost surely, such that $X=Y+\epsilon$ in distribution (Brumelle and Vickson 1975). Therefore, $X$ is noisier than $Y$.

Focusing on base-stock systems, Song (1994a) shows the following results.

LEMMA 5 (SoNG 1994a). If $L_{1} \geqslant_{\mathrm{cx}} L_{2}$, then

(i) $D_{1} \geqslant_{\mathrm{cx}} D_{2}$;

(ii) $G_{1}(y) \geqslant G_{2}(y)$ for all $y$; and

(iii) $G_{1}\left(y_{1}^{*}\right) \geqslant G_{2}\left(y_{2}^{*}\right)$.

Using these results and the relationship between $(r, q)$ and base-stock systems (see (2)), we obtain:

THEOREM 4. Suppose that $L_{1} \geqslant_{\mathrm{cx}} L_{2}$. Then, for any given $(r, q)$ policy, the long-run average cost of system 1 is higher than that of system 2, i.e., $c_{1}(r, q) \geqslant c_{2}(r, q)$. Therefore, the optimal long-run average cost of system 1 is also higher than that of system 2, i.e., $c_{1}\left(r_{1}^{*}, q_{1}^{*}\right) \geqslant c_{2}\left(r_{2}^{*}, q_{2}^{*}\right)$.

To study the effect of lead-time variability on the optimal policy, we shall employ the following variability notion, stronger than the convex order, termed the variability order. (Here, "stronger" means that it implies the convex order.)

For any real function $u(t)$ defined on an ordered set $U$ of the real line, let $S(u)$ be its number of sign changes over the entire set $\mathcal{U}$. (See Shaked and Shanthikumar 1994 for more detail.)

Definition 1. Consider two random variables $X$ and $Y$ having distributions $F$ and $G$ with densities $f$ and $g$. Suppose that $X$ and $Y$ are either both continuous or both discrete. We say $X$ is more variable than $Y$, denoted $X \geqslant_{\text {var }} Y$, if and only if $\mathrm{E}[X]=\mathrm{E}[Y]$ and $\mathrm{S}(f-g)=2$ with sign sequence,,+-+ . That is, $f$ crosses $g$ exactly twice, first from above and then from below.

This is a natural and intuitive property because it compares the spreads of the densities of two random variables. It is the same as the so-called "dangerous order $\left(\geqslant_{D}\right)$ " with identical means (Karlin and Novikoff 1963 and Müller and Stoyan 2002). It is an easier to check and sufficient condition for the convex order (Shaked and Shanthikumar 1994). It is also known that this is a weaker ordering than (i.e., implied by) the "mean preserving spread ( $\left.\geqslant_{\text {MPS }}\right)$ " order commonly used in the economics literature (Müller and Stoyan 2002). Song (1994a) provides many examples of commonly used lead-time distributions that can be compared using $\geqslant_{\text {var }}$, including gamma, uniform, normal, and truncated normal distributions.

The following results are helpful:

Lemma 6 (Song 1994a). If $L_{1} \geqslant{ }_{\text {var }} L_{2}$, then the following properties hold:

(i) $L_{1} \geqslant{ }_{\mathrm{cx}} L_{2}$;

(ii) $G_{1}(y) \geqslant G_{2}(y)$ for all $y$, and $G_{1}\left(y_{1}^{*}\right) \geqslant G_{2}\left(y_{2}^{*}\right)$;

(iii) $\mathrm{S}\left(\Delta G_{1}(y)-\Delta G_{2}(y)\right)=\mathrm{S}\left(\Psi_{1}-\Psi_{2}\right)=1$ with sign sequence,+- . That is, $\Delta G_{1}$ (respectively, $\Psi_{1}$ ) crosses $\Delta G_{2}$ (respectively, $\Psi_{2}$ ) exactly once and the cross is from above;

(iv) $D_{1} \geqslant_{\text {var }} D_{2}$; and

(v) Let $z_{0}$ be the unique (finite) cross point of $\Psi_{1}$ and $\Psi_{2}$ and denote $\theta_{0}=\Psi_{1}\left(z_{0}\right)$. Then, when the ratio $p /(p+h) \geqslant \theta_{0}, y_{1}^{*} \geqslant y_{2}^{*} \geqslant z_{0}$; otherwise, $y_{1}^{*} \leqslant y_{2}^{*} \leqslant z_{0}$.

From Lemma 6 and Theorem 4, we immediately obtain the following results on the cost comparison in $(r, q)$ systems.

THEOREM 5. If $L_{1} \geqslant$ var $L_{2}$, then $c_{1}(r, q) \geqslant c_{2}(r, q)$ for any $(r, q)$. In particular, $c_{1}\left(r_{1}^{*}, q_{1}^{*}\right) \geqslant c_{2}\left(r_{2}^{*}, q_{2}^{*}\right)$.

To study the ordering of policy parameters in $(r, q)$ systems, we first establish the following property:

Lemma 7. Suppose that $L_{1} \geqslant_{\mathrm{var}} L_{2}$. Let $M_{i}(i=1,2)$ be independent uniform $\{n+1, \ldots, n+m\}(n \geqslant 0, m \geqslant 1)$ random variables, which are also independent of $D_{1}$ and $D_{2}$. Then, $D_{1}+M_{1} \geqslant$ var $D_{2}+M_{2}$.

Proof. It follows from Lemma 6 that $D_{1} \geqslant$ var $D_{2}$. This implies that there exist $a$ and $b$ with $0<a<b<\infty$ such that

$\psi_{1}(x) \geqslant \psi_{2}(x)$ for $x \in[0, a)$ and there exists at least one point $x_{1} \in[0, a)$ with $\psi_{1}\left(x_{1}\right)>\psi_{2}\left(x_{1}\right)$,

$\psi_{1}(x) \leqslant \psi_{2}(x)$ for $x \in(a, b)$ and there exists at least one point $x_{2} \in(a, b)$ with $\psi_{1}\left(x_{2}\right)<\psi_{2}\left(x_{2}\right)$,

$\psi_{1}(x) \geqslant \psi_{2}(x)$ for $x \in(b, \infty)$ and there exists at least one point $x_{3} \in(b, \infty)$ with $\psi_{1}\left(x_{3}\right)>\psi_{2}\left(x_{3}\right)$. 
Let $z_{0}>0$ be the cross point of $\Psi_{1}(x)$ and $\Psi_{2}(x)$. Moreover,

$\Psi_{1}(x) \geqslant \Psi_{2}(x)$ for $x \in\left[0, z_{0}\right)$ and there exists at least one point $z_{1} \in\left[0, z_{0}\right)$ with $\Psi_{1}\left(z_{1}\right)>\Psi_{2}\left(z_{1}\right)$,

$\Psi_{1}(x) \leqslant \Psi_{2}(x)$ for $x \in\left(z_{0}, \infty\right)$ and there exists at least

$$
\text { one point } z_{2} \in\left(z_{0}, \infty\right) \text { with } \Psi_{1}\left(z_{2}\right)<\Psi_{2}\left(z_{2}\right) \text {. }
$$

Denote by $\gamma_{i}(\cdot)$ and $\Gamma_{i}(\cdot)$ the density and distribution functions of $D_{i}+M_{i}$, respectively. We have

$\gamma_{i}(x)=\operatorname{Pr}\left\{D_{i}+M_{i}=x\right\}=\sum_{k=n+1}^{n+m} \frac{1}{m} \psi_{i}(x-k)$

and

$\Gamma_{i}(x)=\operatorname{Pr}\left\{D_{i}+M_{i} \leqslant x\right\}=\sum_{k=n+1}^{n+m} \frac{1}{m} \Psi_{i}(x-k)$.

From (19) and (24), there exists $a_{1}$ such that $\gamma_{1}(x) \geqslant \gamma_{2}(x)$ for $x \in\left[0, a_{1}\right)$ and there exists at least one point $\xi_{1} \in\left[0, a_{1}\right)$ with $\gamma_{1}\left(\xi_{1}\right)>\gamma_{2}\left(\xi_{1}\right)$. Let $\hat{a}$ be the largest such $a_{1}$. Then,

$\gamma_{1}(x) \geqslant \gamma_{2}(x)$ for $x \in[0, \hat{a})$ and there exists at least one

$$
\text { point } \xi_{1} \in[0, \hat{a}) \text { with } \gamma_{1}\left(\xi_{1}\right)>\gamma_{2}\left(\xi_{1}\right) \text {. }
$$

Furthermore, using (19) again, we know that $\hat{a} \geqslant a$. On the other hand, by (23) and (25) there exists $\kappa$ such that

$\Gamma_{1}(\kappa)<\Gamma_{2}(\kappa)$.

This implies that there exists $b_{1}<\infty$ such that $\gamma_{1}\left(b_{1}\right)<$ $\gamma_{2}\left(b_{1}\right)$. Therefore, $\hat{a}<\infty$ in (26). Now choose the largest $\hat{b}$ such that

$\gamma_{1}(x) \leqslant \gamma_{2}(x)$ for $x \in(\hat{a}, \hat{b})$ and there exists at least one point $\xi_{2} \in(\hat{a}, \hat{b})$ with $\gamma_{1}\left(\xi_{2}\right)<\gamma_{2}\left(\xi_{2}\right)$.

Using (20), $\hat{b} \geqslant b$. By $\lim _{y \rightarrow \infty} \Gamma_{1}(y)=\lim _{y \rightarrow \infty} \Gamma_{2}(y)=1$, (27), and (28), we have $\hat{b}<\infty$. This implies

$\gamma_{1}(x) \geqslant \gamma_{2}(x)$ for $x \in(\hat{b}, \infty)$ and there exists at least one point $\xi_{3} \in(\hat{b}, \infty)$ with $\gamma_{1}\left(\xi_{3}\right)>\gamma_{2}\left(\xi_{3}\right)$.

Combining (26), (28), and (29), we obtain the desired result.

We now show the following generalization of Lemma 6(v).

THEOREM 6. Suppose that $L_{1} \geqslant_{\mathrm{var}} L_{2}$.

(i) For any fixed $q, C_{1}(q, y) / q$ crosses $C_{2}(q, y) / q$ exactly once at a finite point $z(q)$, and the cross is from above. Let $\theta(q)=C_{1}(q, z(q)) / q$. Then, when the ratio $p /(p+h)>\theta(q), r_{1}^{*}(q) \geqslant r_{2}^{*}(q) \geqslant z(q)$; otherwise, $r_{1}^{*}(q) \leqslant r_{2}^{*}(q) \leqslant z(q) ;$ and (ii) $z(q)$ is nonincreasing, that is, $z(q+1) \leqslant z(q)$.

(Note that $C_{i}(1, y)=\Psi_{i}(y+1)$, so $z(1)=z_{0}-1$ and $\theta(1)=\theta_{0}$.)

Proof. Part (i) follows from Lemmas 1, 6(v), and 7. To prove Part (ii), noting that

$z(q)=\inf \left\{y: C_{1}(q, y) \leqslant C_{2}(q, y)\right\}$,

it suffices to prove that if for some $y$,

$\sum_{x=1}^{q} \Psi_{1}(y+x) \leqslant \sum_{x=1}^{q} \Psi_{2}(y+x)$,

then

$\sum_{x=1}^{q+1} \Psi_{1}(y+x) \leqslant \sum_{x=1}^{q+1} \Psi_{2}(y+x)$.

Note that if (30) holds, then by (22) and (23) we must have $y+q \geqslant z_{0}$. Hence, $y+q+1>z_{0}$. From (22) and (30), we then have (31), proving Part (ii).

Theorem 6 indicates that for any given $q$, the effect of lead-time variability on the optimal reorder point resembles that in the base-stock system. Property (ii) further sheds light on the strength of this effect over different values of $q$, everything else being equal. In particular, from the nondecreasing property of $z(q)$, we see that, for any given $q$, a more variable lead time requires a smaller reorder point. Then, for an even bigger $q$, the same order is preserved. In other words, a bigger $q$ provides less incentive for a bigger reorder point to hedge against the same level of supply uncertainty.

Although we have a good understanding of systems with a fixed order quantity, the behavior of the optimal policy parameters turns out to be more subtle. The following theorem summarizes the results on the optimal reorder point and order-up-to level. Its proof is in the appendix.

THEOREM 7. Under the assumption of $L_{1} \geqslant_{\mathrm{var}} L_{2}$, we have:

(i) if $r_{1}^{*}>r_{2}^{*}$, then $r_{1}^{*}+q_{1}^{*} \geqslant r_{2}^{*}+q_{2}^{*}$; and

(ii) if $r_{1}^{*}<r_{2}^{*}$ and $z_{0} \notin\left(r_{2}^{*}, r_{2}^{*}+q_{2}^{*}\right)$, then $r_{1}^{*}+q_{1}^{*} \leqslant r_{2}^{*}+$ $q_{2}^{*}$, where $z_{0}$ is the cross point of $\Psi_{1}$ and $\Psi_{2}$.

The following example shows that if the second condition in Theorem 7, Part (ii) does not hold, then the result may not hold. That is, if $r_{1}^{*}<r_{2}^{*}$ and $z_{0} \in\left(r_{2}^{*}, r_{2}^{*}+q_{2}^{*}\right)$, then we may not have $r_{1}^{*}+q_{1}^{*} \leqslant r_{2}^{*}+q_{2}^{*}$. (Note that from Lemma 6(iv), the study of a more variable lead time reduces to that of a more variable lead-time demand. For simplicity, the examples in the the remainder of the section focus on the variability lead-time demand distributions, with the understanding that the same insights apply to the lead-time variability.) The data of the example are drawn from Zheng (1992). 
ExAmple 2. Consider

$\psi_{1}(k)=\frac{50^{k}}{k !} e^{-50}, \quad k=0,1, \ldots$,

$\psi_{2}(k)= \begin{cases}1, & \text { if } k=50 \\ 0, & \text { otherwise }\end{cases}$

$p=25, \quad h=10$.

With these probability density functions, clearly, $D_{1} \geqslant_{\text {var }} D_{2}$. Furthermore, $\mathrm{E} D_{1}=\mathrm{E} D_{2}=50$ and $z_{0}=50$. We have

$\begin{array}{rrrrr}K & r_{1}^{*} & r_{2}^{*} & q_{1}^{*} & q_{2}^{*} \\ 1 & 50 & 48 & 7 & 4 \\ 5 & 48 & 47 & 12 & 8 \\ 25 & 44 & 44 & 23 & 19 \\ 100 & 38 & 39 & 40 & 37 \\ 1,000 & 15 & 16 & 120 & 118\end{array}$

Here, we observe that for certain values of $K, r_{1}^{*}>r_{2}^{*}$, whereas for other values of $K, r_{1}^{*}<r_{2}^{*}$. In all cases, $z_{0} \in$ $\left(r_{2}^{*}, r_{2}^{*}+q_{2}^{*}\right)$ and $r_{1}^{*}+q_{1}^{*}>r_{2}^{*}+q_{2}^{*}$.

Finally, we discuss the behavior of the optimal order quantity. In an extreme case, assume that $D_{2}$ is a constant, denoted by $D_{d}$, where the subscript " $d$ " represents "deterministic" as in Zheng (1992). Then, for any nonnegative random $D_{1}$ with $\mathrm{E}\left[D_{1}\right]=D_{d}, D_{1} \geqslant{ }_{\text {var }} D_{2}$. According to Theorem 2 of Zheng (1992), we have $q_{1}^{*} \geqslant q_{2}^{*}$. This special case may naturally inspire a conjecture that for any pair of lead-time demand variables satisfying $D_{1} \geqslant$ var $D_{2}$, we have $q_{1}^{*} \geqslant q_{2}^{*}$. However, this is not necessarily the case, as shown in the following example.

Example 3. Consider two $(r, q)$ systems with $\lambda=1$, $h=45$, and $p=55$. Suppose that the lead-time demands $D_{1}$ and $D_{2}$ have densities

$\psi_{1}(x)= \begin{cases}\frac{1}{150}, & x \in\{1,2, \ldots, 120\}, \\ \frac{1}{160}, & x \in\{145,146, \ldots, 176\}, \\ 0, & \text { otherwise }\end{cases}$
$\psi_{2}(x)= \begin{cases}\frac{1}{160}, & x \in\{1,2, \ldots, 160\}, \\ 0, & \text { otherwise. }\end{cases}$

Then, $\mathrm{E} D_{1}=\mathrm{E} D_{2}=80.5$ and $D_{1} \geqslant_{\text {var }} D_{2}$. With these settings, we have:

(i) $y_{1}^{*}=83<y_{2}^{*}=88$. Thus, for the system with a more variable lead-time demand, the optimal base-stock level is actually lower. This can be explained by the fact that the backorder penalty cost is not very high, compared to the holding cost;

(ii) For $K=24$, we have $r_{1}^{*}=79, q_{1}^{*}=7, r_{2}^{*}=84$, and $q_{2}^{*}=8$. Here, $q_{1}^{*}<q_{2}^{*}$; and (iii) For $K=60$, we have $r_{1}^{*}=77, q_{1}^{*}=11, r_{2}^{*}=83$, and $q_{2}^{*}=10$. Here, $q_{1}^{*}>q_{2}^{*}$.

This example shows that for different values of $K, q_{i}^{*}$ can be ordered in either way under the variability order, even though $D_{i}$ and other cost parameters stay the same. Thus, the precise effect of variability on the optimal order quantity depends on the magnitude of the fixed ordering cost $K$.

\section{Other Demand Processes and Effect of Demand Variability}

\subsection{Compound-Poisson and Renewal Demand Processes}

Suppose now that the demand process is a compoundPoisson process. That is, demand epochs occur according to a Poisson process with rate $\lambda$, and at each such epoch there is a demand for a batch of random size $Z$. The batches form a sequence of i.i.d., positive integer random variables with the same distribution as $Z$. Because the demand size can be greater than one, the inventory position at a demand epoch may overshoot the reorder point $r$, the order triggered by this demand will need to comprise multiple lots of size $q$ to bring the inventory position above $r$. For this reason, the $(r, q)$ policy in the nonunit demand environment is often termed the $(r, n q)$ policy, where $n$ represents the smallest number of lots needed to bring the inventory position above $r$. We assume that a fixed cost $K$ is charged for each lot in the order (such as each truckload). Then, the cost function $c_{i}(r, q)$ in (2) remains the same, except that the constant $K$ should be replaced by $K E[Z]$. Correspondingly, the optimality conditions and the algorithms above all apply.

From Song (1994a), we have $L_{1} \geqslant_{\mathrm{st}} L_{2} \Rightarrow D_{1} \geqslant{ }_{\mathrm{st}} D_{2}$ and $L_{1} \geqslant_{\text {cx }} L_{2} \Rightarrow D_{1} \geqslant_{\text {cx }} D_{2}$. Thus, all results in the previous sections concerning these stochastic orders still hold here. In addition, if the probability mass function of $Z$ is of a Polya frequency of order $3\left(\mathrm{PF}_{3}\right)$, including the Poisson, geometric, binomial, and negative binomial densities, then Song (1994a) shows that $L_{1} \geqslant_{\text {var }} L_{2} \Rightarrow D_{1} \geqslant_{\text {var }} D_{2}$, and therefore all results regarding the variability $\left(\geqslant_{v a r}\right)$ order apply.

As we have observed in earlier sections, all the key results of this paper hold if the stochastic comparisons are based on the lead-time demand distributions rather than on the lead-time distributions. Furthermore, if the comparisons are conducted on this basis, the compound-Poisson assumption is not necessary as long as the fixed ordering cost is charged on a per-lot basis. In particular, the demand process can be a renewal process; a stochastically larger (respectively, more variable) lead-time demand may result from a stochastically smaller (respectively, more variable) interdemand but a fixed lead-time distribution, or from a stochastically larger (respectively, more variable) lead time but a fixed interdemand distribution, or from both. 


\subsection{Continuous-Demand Process and Normal Lead Time Demand}

Suppose that the product in stock is infinitely divisible or of high volume so that a continuous-demand process is more appropriate. Then, the expected long-run average cost can be expressed as

$c_{i}(r, q)=\frac{\lambda K+\int_{r}^{r+q} G_{i}(y) \mathrm{d} y}{q}$,

with $G_{i}(y)=h \mathrm{E}\left[\left(y-D_{i}\right)^{+}\right]+p \mathrm{E}\left[\left(D_{i}-y\right)\right]^{+}$. (For conditions such that this expression is valid, see Zheng 1992.) According to Zheng (1992), for any given $q, r=$ $r_{i}^{*}(q)$ is obtained if the chord connecting $\left(r, G_{i}(r)\right)$ and $\left(r+q, G_{i}(r+q)\right)$ is horizontal, and $q=q_{i}^{*}$ is obtained if the area between the chord and $G_{i}(\cdot)$ is equal to $\lambda K$. Formally, this intuitive result can be stated as:

Lemma 8 (Zheng 1992). (i) For any fixed $q, G_{i}\left(r_{i}^{*}(q)\right)=$ $G_{i}\left(r_{i}^{*}(q)+q\right)$

(ii) $q G_{i}\left(r_{i}^{*}(q)\right)-\int_{r_{i}^{*}(q)}^{r_{*}^{*}(q)+q} G_{i}(y) \mathrm{d} y$ is an increasing convex function. $q=q_{i}^{*}$ if and only if $\lambda K=q G_{i}\left(r_{i}^{*}(q)\right)-$ $\int_{r_{i}^{*}(q)}^{r_{i}^{*}(q)+q} G_{i}(y) \mathrm{d} y$.

Using the above geometric representation of the optimality conditions and similar arguments in the discrete demand case, it can be shown that all the results in the previous sections on the effect of the lead-time demand $D_{i}$ equally apply here.

If the lead-time demand is well approximated by a normal distribution with mean $\mu$ and standard deviation $\sigma$, we obtain even stronger results. The normal distribution is perhaps the most commonly used lead-time demand distribution in the literature and in practice; see Tyworth and O'Neill (1997). Zipkin (2000) illustrates through some numerical examples that the optimal order quantity $q^{*}$ is increasing in $\sigma$ (see his Figure 6.5.3). In Theorem 8 below we show analytically that this is true if the fixed ordering cost $K$ is above a threshold. In addition, in Theorem 9 we establish the monotonicity of $r^{*}+q^{*}$ with respect to $\sigma$.

First, we introduce some notation. Let $\phi(\cdot)$ and $\Phi(\cdot)$ be the standard normal density and cumulative distribution functions, respectively. Denote $\Phi^{0}(z)=1-\Phi(z)$ and

$\Phi^{1}(z)=\int_{z}^{+\infty} \Phi^{0}(x) \mathrm{d} x=-z \Phi^{0}(z)+\phi(z)$.

Let

$z_{y}=\frac{y-\mu}{\sigma}$.

Note that

$\phi^{\prime}(z)=-z \phi(z), \quad\left[\Phi^{1}(z)\right]^{\prime}=-\Phi^{0}(z)$.

Then,

$G(y)=h(y-\mu)+(p+h) \sigma \Phi^{1}\left(z_{y}\right)$,

$G^{\prime}(y)=(p+h) \Phi\left(z_{y}\right)-p$,

$G^{\prime \prime}(y)=\frac{p+h}{\sigma} \phi\left(z_{y}\right)$.
Also, because $\partial z_{y} / \partial \sigma=-z_{y} / \sigma$, we have

$\frac{\partial G(y)}{\partial \sigma}=(p+h) \phi\left(z_{y}\right)$,

$\frac{\partial G^{\prime}(y)}{\partial \sigma}=-\frac{p+h}{\sigma} z_{y} \phi\left(z_{y}\right)=\frac{p+h}{\sigma} \phi^{\prime}\left(z_{y}\right)$.

Now, we show the variability effect on $q^{*}$.

Theorem 8. Suppose that $D_{i} \sim N\left(\mu, \sigma_{i}^{2}\right)$ and $D_{1} \geqslant$ var $D_{2}$. Then, there exists $K_{0}$ such that for all $K>K_{0}, q_{1}^{*}>q_{2}^{*}$. Equivalently, suppose that the lead-time demand $D \sim$ $N\left(\mu, \sigma^{2}\right)$. Then, $\partial q^{*} / \partial \sigma \geqslant 0$ if $K \geqslant K_{0}$.

Proof. Let $r^{*}$ and $q^{*}$ be the optimal reorder point and order quantity for the demand with normal distribution $N\left(\mu, \sigma^{2}\right)$. For a simpler exposition, in the following, $r^{*}$ and $q^{*}$ are simply written as $r$ and $q$. To prove the theorem, it suffices to prove $\partial q / \partial \sigma>0$.

Recall that the optimal reorder point $r$ and order quantity $q$ satisfy

$\int_{r}^{r+q}(G(r)-G(y)) \mathrm{d} y=\lambda K$,

$G(r+q)=G(r)$.

Using (36), we have

$\int_{r}^{r+q}(G(r)-G(y)) \mathrm{d} y=\int_{r}^{r+q} y G^{\prime}(y) \mathrm{d} y$.

Thus, by (35), we have

$\int_{r}^{r+q} y G^{\prime}(y) \mathrm{d} y=\lambda K$.

Taking the derivative with respect to $\sigma$ on both sides of the above equality yields

$$
\begin{aligned}
(r & +q) G^{\prime}(r+q) \frac{\partial q}{\partial \sigma}+\left((r+q) G^{\prime}(r+q)-r G^{\prime}(r)\right) \frac{\partial r}{\partial \sigma} \\
& =-\int_{r}^{r+q} y \frac{\partial G^{\prime}(y)}{\partial \sigma} \mathrm{d} y .
\end{aligned}
$$

Note that $r, q$, and $G$ are all functions of $\sigma$. Taking the derivative with respect to $\sigma$ on both sides of (36) and then rearranging the terms, we obtain

$$
\begin{aligned}
& G^{\prime}(r+q) \frac{\partial q}{\partial \sigma}+\left(G^{\prime}(r+q)-G^{\prime}(r)\right) \frac{\partial r}{\partial \sigma} \\
& =-\frac{\partial(G(r+q)-G(r))}{\partial \sigma} .
\end{aligned}
$$

We can rewrite (37) and (38) as

$A\left(\begin{array}{l}\partial q / \partial \sigma \\ \partial r / \partial \sigma\end{array}\right)=\left(\begin{array}{l}-b_{1} \\ -b_{2}\end{array}\right)$, 
where

$A=\left(\begin{array}{cc}(r+q) G^{\prime}(r+q) & (r+q) G^{\prime}(r+q)-r G^{\prime}(r) \\ G^{\prime}(r+q) & G^{\prime}(r+q)-G^{\prime}(r)\end{array}\right)$

and

$b_{1}=\int_{r}^{r+q} y \frac{\partial G^{\prime}(y)}{\partial \sigma} \mathrm{d} y, \quad b_{2}=\frac{\partial(G(r+q)-G(r))}{\partial \sigma}$.

Note that $|A|=-q G^{\prime}(r+q) G^{\prime}(r)>0$. Therefore, the above system of linear equations has a unique solution, and the sign of $\partial q / \partial \sigma$ is determined by the sign of

$A_{1}=\left|\begin{array}{cc}-b_{1} & (r+q) G^{\prime}(r+q)-r G^{\prime}(r) \\ -b_{2} & G^{\prime}(r+q)-G^{\prime}(r)\end{array}\right|$.

Thus, what remains to show is $A_{1}>0$.

From (34), we have

$$
\begin{aligned}
b_{1}= & \frac{p+h}{\sigma} \int_{r}^{r+q} y \phi^{\prime}\left(z_{y}\right) \mathrm{d} y \\
= & (p+h) \int_{r}^{r+q} y \mathrm{~d} \phi\left(z_{y}\right) \\
= & (p+h)\left((r+q) \phi\left(z_{r+q}\right)-r \phi\left(z_{r}\right)\right. \\
& \left.\quad-\sigma\left[\Phi\left(z_{r+q}\right)-\Phi\left(z_{r}\right)\right]\right) .
\end{aligned}
$$

Using (33), we have $b_{2}=(p+h)\left(\phi\left(z_{r+q}\right)-\phi\left(z_{r}\right)\right)$. By (32),

$$
\begin{aligned}
& (r+q) G^{\prime}(r+q)-r G^{\prime}(r) \\
& \quad=(p+h)\left[(r+q) \Phi\left(z_{r+q}\right)-r \Phi\left(z_{r}\right)\right]-p q
\end{aligned}
$$

and $G^{\prime}(r+q)-G^{\prime}(r)=(p+h)\left[\Phi\left(z_{r+q}\right)-\Phi\left(z_{r}\right)\right]$. From these, we can show that

$$
\begin{aligned}
(p+h)^{-1} A_{1}= & \sigma(p+h)\left[\Phi\left(z_{r+q}\right)-\Phi\left(z_{r}\right)\right]^{2} \\
& -q\left[\phi\left(z_{r}\right) G^{\prime}(r+q)-\phi\left(z_{r+q}\right) G^{\prime}(r)\right] \\
\geqslant & \sigma(p+h)\left[\Phi\left(z_{r+q}\right)-\Phi\left(z_{r}\right)\right]^{2} \\
& -(p+h) q \phi\left(z_{r}\right) \Phi\left(z_{r+q}\right)-p q \phi\left(z_{r+q}\right) .
\end{aligned}
$$

Note that from Lemmas 3 and 6 of Zheng (1992), $\lim _{K \rightarrow+\infty} r=-\infty$ and $\lim _{K \rightarrow+\infty}[r+q]=+\infty$. Therefore, $\lim _{K \rightarrow+\infty} \sigma\left[\Phi\left(z_{r+q}\right)-\Phi\left(z_{r}\right)\right]^{2}=\sigma$. We now proceed to show

$\lim _{K \rightarrow+\infty}\left[q \phi\left(z_{r}\right) \Phi\left(z_{r+q}\right)\right]=0$ and $\lim _{K \rightarrow+\infty} q \phi\left(z_{r+q}\right)=0$

so that $\lim _{K \rightarrow+\infty} A_{1} \geqslant(p+h)^{2} \sigma>0$, and therefore the proof is complete.

Recall that $G(r)=G(r+q)$. Differentiating the equality with respect to $q$, we have

$$
G^{\prime}(r) \frac{\partial r}{\partial q}=G^{\prime}(r+q)\left(1+\frac{\partial r}{\partial q}\right) .
$$

Therefore,

$\lim _{K \rightarrow \infty} \frac{\partial r}{\partial q}=\lim _{K \rightarrow \infty} \frac{-G^{\prime}(r+q)}{G^{\prime}(r+q)-G^{\prime}(r)}=\frac{-h}{p+h}$.

Applying L'Hôpital's rule, we obtain

$$
\begin{aligned}
& \lim _{K \rightarrow \infty} q e^{-z_{r}^{2} / 2} \\
& \quad=\lim _{K \rightarrow \infty} \frac{q}{e^{(r-\mu)^{2} /\left(2 \sigma^{2}\right)}} \\
& \quad=\lim _{K \rightarrow \infty} \frac{1}{e^{(r-\mu)^{2} /\left(2 \sigma^{2}\right)}\left((r-\mu) / \sigma^{2}\right)(\partial r / \partial q)}=0 \\
& \lim _{K \rightarrow \infty} q e^{-z_{r+q}^{2} / 2} \\
& \quad=\lim _{K \rightarrow \infty} \frac{q}{e^{(r+q-\mu)^{2} /\left(2 \sigma^{2}\right)}} \\
& \quad=\lim _{K \rightarrow \infty} \frac{1}{e^{(r+q-\mu)^{2} /\left(2 \sigma^{2}\right)}\left((r+q-\mu) / \sigma^{2}\right)(1+\partial r / \partial q)}=0,
\end{aligned}
$$

proving (39).

Finally, we establish the variability effect on $r^{*}+q^{*}$. The proof is in the appendix.

TheOrem 9. Suppose that $p>h$ and the lead-time demand $D_{i} \sim N\left(\mu, \sigma_{i}^{2}\right)$. Then, $D_{1} \geqslant_{\mathrm{var}} D_{2}$ implies $r_{1}^{*}+q_{1}^{*}>r_{2}^{*}+q_{2}^{*}$. Equivalently, suppose that the lead-time demand $D \sim N\left(\mu, \sigma^{2}\right)$. Then, $r^{*}+q^{*}$ is increasing with respect to $\sigma$.

\section{Appendix}

Proof of Lemma 2. Note that for any fixed $y$,

$$
\begin{aligned}
& \frac{1}{q+1} C_{i}(q+1, y)-\frac{1}{q} C_{i}(q, y) \\
& =\frac{1}{q+1} \sum_{x=1}^{q+1} \Psi_{i}(y+x)-\frac{1}{q} \sum_{x=1}^{q} \Psi_{i}(y+x) \\
& =\frac{1}{q+1} \sum_{x=1}^{q+1} \Psi_{i}(y+x)-\frac{1}{q+1}\left(1+\frac{1}{q}\right) \sum_{x=1}^{q} \Psi_{i}(y+x) \\
& =\frac{1}{q+1} \Psi_{i}(y+q+1)-\frac{1}{q+1} \frac{1}{q} \sum_{x=1}^{q} \Psi_{i}(y+x) \\
& =\frac{1}{q+1} \frac{1}{q} \sum_{x=1}^{q}\left[\Psi_{i}(y+q+1)-\Psi_{i}(y+x)\right] \\
& \geqslant 0 .
\end{aligned}
$$

Thus, $C_{i}(q, y) / q$ increases in $q$. The monotonicity concerning $r_{i}^{*}(q)$ follows from this property and Lemma 1 .

Proof of Theorem 3. From Song (1994a), for any y,

$-h \lambda\left[\mathrm{E} L_{1}-\mathrm{E} L_{2}\right] \leqslant G_{1}(y)-G_{2}(y) \leqslant p \lambda\left[\mathrm{E} L_{1}-\mathrm{E} L_{2}\right]$.

Note that

$c_{1}(r, q)-c_{2}(r, q)=\frac{\sum_{j=r+1}^{r+q}\left[G_{1}(j)-G_{2}(j)\right]}{q}$. 
Therefore, (18) directly follows from (40). By

$c_{1}\left(r_{1}^{*}, q_{1}^{*}\right)-c_{2}\left(r_{2}^{*}, q_{2}^{*}\right) \leqslant c_{1}\left(r_{2}^{*}, q_{2}^{*}\right)-c_{2}\left(r_{2}^{*}, q_{2}^{*}\right)$,

$c_{1}\left(r_{1}^{*}, q_{1}^{*}\right)-c_{2}\left(r_{2}^{*}, q_{2}^{*}\right) \geqslant c_{1}\left(r_{1}^{*}, q_{1}^{*}\right)-c_{2}\left(r_{1}^{*}, q_{1}^{*}\right)$,

we know that $c_{1}\left(r_{1}^{*}, q_{1}^{*}\right)-c_{2}\left(r_{2}^{*}, q_{2}^{*}\right)$ has the same bounds as $c_{1}(r, q)-c_{2}(r, q)$.

Proof of Theorem 7. We shall show Part (i) only. The proof of Part (ii) can be done similarly.

Suppose, contrariwise, that

$r_{1}^{*}+q_{1}^{*}<r_{2}^{*}+q_{2}^{*}$

Using $\Delta G_{i}(y)=(p+h) \Psi_{i}(y)-p$ and the definition of $z_{0}$,

$\Delta G_{1}(y) \leqslant \Delta G_{2}(y)$ for $y \geqslant z_{0}$,

$\Delta G_{1}(y) \geqslant \Delta G_{2}(y)$ for $y<z_{0}$.

Using (6), for any $q_{i}<q_{i}^{*}$ and $\hat{q}_{i} \geqslant q_{i}^{*}$,

$$
\begin{aligned}
q_{i} \cdot & G_{i}\left(y_{i}^{q_{i}+1}\right)-\sum_{k=1}^{q_{i}} G_{i}\left(y_{i}^{k}\right) \\
& <\lambda K \leqslant \hat{q}_{i} \cdot G_{i}\left(y_{i}^{\hat{q}_{i}+1}\right)-\sum_{k=1}^{\hat{q}_{i}} G_{i}\left(y_{i}^{k}\right) .
\end{aligned}
$$

Let

$q_{2}^{0}=\min \left\{q_{2}:\left\{r_{1}^{*}+1, \ldots, r_{1}^{*}+q_{1}^{*}\right\} \subseteq\left\{y_{2}^{1}, \ldots, y_{2}^{q_{2}}\right\}\right\}$

Clearly,

$y_{2}^{q_{2}^{0}}=r_{1}^{*}+1 \quad$ or $y_{2}^{q_{2}^{0}}=r_{1}^{*}+q_{1}^{*}$.

In view of (41), $r_{2}^{*}+1<r_{1}^{*}+1 \leqslant r_{1}^{*}+q_{1}^{*}<r_{2}^{*}+q_{2}^{*}$. By (4), we have

$\left\{y_{2}^{1}, \ldots, y_{2}^{q_{2}^{0}}\right\} \subseteq\left\{r_{2}^{*}+1, \ldots, r_{2}^{*}+q_{2}^{*}\right\}$,

$q_{1}^{*} \leqslant q_{2}^{0} \leqslant q_{2}^{*}$.

The remainder of the proof is divided into the following three cases:

Case 1. $z_{0} \leqslant r_{2}^{*}$;

Case 2. $z_{0}>r_{2}^{*}+q_{2}^{*}$; and

Case 3. $r_{2}^{*}<z_{0} \leqslant r_{2}^{*}+q_{2}^{*}$.

Consider Case 1. We first argue that, under this case,

$r_{1}^{*}+q_{1}^{*}+1 \notin\left\{y_{2}^{1}, \ldots, y_{2}^{q_{2}^{0}}\right\}$.

Suppose this is not true. Then, it follows from the definition of $q_{2}^{0}$ that $r_{1}^{*}+q_{1}^{*}+1 \neq y_{2}^{q_{2}^{0}}$. Note that (see (4))

$G_{2}\left(y_{2}^{1}\right) \leqslant G_{2}\left(y_{2}^{2}\right) \leqslant \cdots \leqslant G_{2}\left(y_{2}^{q_{2}^{0}}\right)$.
In view of $r_{1}^{*}+q_{1}^{*}+1 \in\left\{y_{2}^{1}, \ldots, y_{2}^{q_{2}^{0}}\right\}$ and the definition of $q_{2}^{0}$, if $r_{1}^{*}+q_{1}^{*}+1 \neq y_{2}^{q_{2}^{0}}$, then

$G_{2}\left(r_{1}^{*}+q_{1}^{*}+1\right)<G_{2}\left(r_{1}^{*}+1\right)$.

On the other hand,

$G_{1}\left(r_{1}^{*}+q_{1}^{*}+1\right) \geqslant G_{1}\left(r_{1}^{*}+1\right)$.

By $r_{1}^{*}+1>z_{0}$ and $r_{1}^{*}+q_{1}^{*}+1>z_{0}$, we know that (46) and (47) contradict (42). Therefore, we have (45).

Let

$\widehat{q}_{2}^{0}=\inf \left\{q_{2}:\left\{r_{1}^{*}+1, \ldots, r_{1}^{*}+q_{1}^{*}, r_{1}^{*}+q_{1}^{*}+1\right\} \subseteq\left\{y_{2}^{1}, \ldots, y_{2}^{q_{2}}\right\}\right\}$.

According to $r_{2}^{*}+1<r_{1}^{*}+1<r_{1}^{*}+q_{1}^{*}<r_{2}^{*}+q_{2}^{*}$ and (4), we have

$\hat{q}_{2}^{0} \leqslant q_{2}^{*} \quad$ and $\quad \hat{q}_{2}^{0}-1 \geqslant q_{1}^{*}$.

Again using (4), we have $G_{1}\left(r_{1}^{*}+q_{1}^{*}+1\right) \geqslant G_{1}\left(y_{1}^{q_{1}^{*}+1}\right)$. Hence, by (44),

$\lambda K \leqslant q_{1}^{*} \cdot G_{1}\left(r_{1}^{*}+q_{1}^{*}+1\right)-\sum_{k=1}^{q_{1}^{*}} G_{1}\left(y_{1}^{k}\right)$.

By (44) and (48),

$$
\begin{aligned}
\lambda K & >\left(\hat{q}_{2}^{0}-1\right) \cdot G_{2}\left(y_{2}^{\hat{q}_{2}^{0}}\right)-\sum_{k=1}^{\hat{q}_{2}^{0}-1} G_{2}\left(y_{2}^{k}\right) \\
& \geqslant\left(\hat{q}_{2}^{0}-1\right) \cdot G_{2}\left(r_{1}^{*}+q_{1}^{*}+1\right)-\sum_{k=1}^{\hat{q}_{2}^{0}-1} G_{2}\left(y_{2}^{k}\right) .
\end{aligned}
$$

On the other hand, by (42) and (48), we have

$$
\begin{gathered}
\left(\hat{q}_{2}^{0}-1\right) \cdot G_{2}\left(r_{1}^{*}+q_{1}^{*}+1\right)-\sum_{k=1}^{\hat{q}_{2}^{0}-1} G_{2}\left(y_{2}^{k}\right) \\
\geqslant q_{1}^{*} \cdot G_{1}\left(r_{1}^{*}+q_{1}^{*}+1\right)-\sum_{k=1}^{q_{1}^{*}} G_{1}\left(y_{1}^{k}\right),
\end{gathered}
$$

which contradicts (49) and (50). Thus, Case 1 is proved.

Next, we consider Case 2. Similar to (45), we can show that under Case 2,

$r_{1}^{*} \notin\left\{y_{2}^{1}, \ldots, y_{2}^{q_{2}^{0}}\right\}$.

Define

$\tilde{q}_{2}^{0}=\inf \left\{q_{2}:\left\{r_{1}^{*}, r_{1}^{*}+1, \ldots, r_{1}^{*}+q_{1}^{*}\right\} \subseteq\left\{y_{2}^{1}, \ldots, y_{2}^{q_{2}}\right\}\right\}$.

According to $r_{2}^{*}+1<r_{1}^{*}+1<r_{1}^{*}+q_{1}^{*}<r_{2}^{*}+q_{2}^{*}$ and (4), we have

$\tilde{q}_{2}^{0} \leqslant q_{2}^{*} \quad$ and $\quad \tilde{q}_{2}^{0}-1 \geqslant q_{1}^{*}$.

By (43) and (51), we have

$\left(\tilde{q}_{2}^{0}-1\right) \cdot G_{2}\left(r_{1}^{*}\right)-\sum_{k=1}^{\tilde{q}_{2}^{0}-1} G_{2}\left(y_{2}^{k}\right) \geqslant q_{1}^{*} \cdot G_{1}\left(r_{1}^{*}\right)-\sum_{k=1}^{q_{1}^{*}} G_{1}\left(y_{1}^{k}\right)$,

which contradicts (44). Thus, Case 2 is proved. 
Finally, we go to Case 3. There are three subcases, namely,

Subcase 3.1. $r_{2}^{*}+1 \leqslant z_{0} \leqslant r_{1}^{*}+1$;

Subcase 3.2. $r_{1}^{*}+q_{1}^{*} \leqslant z_{0} \leqslant r_{2}^{*}+q_{2}^{*}$; and

Subcase 3.3. $r_{1}^{*}+1<z_{0}<r_{1}^{*}+q_{1}^{*}$.

Along the lines of Cases 1 and 2, we can obtain contradictions for the first two subcases. Hence, it is sufficient to consider Subcase 3.3. Let

$\bar{q}_{2}^{0}=\inf \left\{q_{2}:\left\{r_{1}^{*}, \ldots, r_{1}^{*}+q_{1}^{*}, r_{1}^{*}+q_{1}^{*}+1\right\} \subseteq\left\{y_{2}^{1}, \ldots, y_{2}^{q_{2}}\right\}\right\}$.

Clearly,

$y_{2}^{\bar{q}_{2}^{0}}=r_{1}^{*} \quad$ or $\quad y_{2}^{\bar{q}_{2}^{0}}=r_{1}^{*}+q_{1}^{*}+1$

Furthermore, under this subcase, we have

$r_{2}^{*}+1<r_{1}^{*}+1<z_{0}<r_{1}^{*}+q_{1}^{*}<r_{2}^{*}+q_{2}^{*}$.

This and (4) imply that

$\bar{q}_{2}^{0} \leqslant q_{2}^{*}$

It follows from (44) that

$$
\begin{aligned}
\lambda K & \leqslant q_{1}^{*} \cdot G_{1}\left(y_{1}^{q_{1}^{*}+1}\right)-\sum_{k=1}^{q_{1}^{*}} G_{1}\left(y_{1}^{k}\right) \\
& =q_{1}^{*} \cdot \min \left\{G_{1}\left(r_{1}^{*}\right), G_{1}\left(r_{1}^{*}+q_{1}^{*}+1\right)\right\}-\sum_{k=1}^{q_{1}^{*}} G_{1}\left(y_{1}^{k}\right) .
\end{aligned}
$$

By (44) and (53), if the first equality of (52) holds (i.e., $\left.y_{2}^{q_{2}^{0}}=r_{1}^{*}\right)$, then

$$
\begin{aligned}
\lambda K & >\left(\bar{q}_{2}^{0}-1\right) \cdot G_{2}\left(y_{2}^{\bar{q}_{2}^{0}}\right)-\sum_{k=1}^{\bar{q}_{2}^{0}-1} G_{2}\left(y_{2}^{k}\right) \\
& =\left(\bar{q}_{2}^{0}-1\right) \cdot G_{2}\left(r_{1}^{*}\right)-\sum_{k=1}^{\bar{q}_{2}^{0}-1} G_{2}\left(y_{2}^{k}\right),
\end{aligned}
$$

and if the second equality of (52) holds (i.e., $y_{2}^{\bar{q}_{2}^{0}}=$ $\left.r_{1}^{*}+q_{1}^{*}+1\right)$, then

$\lambda K>\left(\bar{q}_{2}^{0}-1\right) \cdot G_{2}\left(r_{1}^{*}+q_{1}^{*}+1\right)-\sum_{k=1}^{\bar{q}_{2}^{0}-1} G_{2}\left(y_{2}^{k}\right)$.

Define

$\left\{\tilde{y}_{1}^{1}, \ldots, \tilde{y}_{1}^{\tilde{n}_{1}}\right\}=\left\{r_{1}^{*}+1, \ldots, z_{0}\right\} \quad$ with $\tilde{y}_{1}^{1}<\cdots<\tilde{y}_{1}^{\tilde{k}_{1}}$,

$\left\{\hat{y}_{1}^{1}, \ldots, \hat{y}_{1}^{\hat{k}_{1}}\right\}=\left\{z_{0}+1, \ldots, r_{1}^{*}+q_{1}^{*}\right\} \quad$ with $\hat{y}_{1}^{1}<\cdots<\hat{y}_{1}^{\hat{k}_{1}}$,

$\left\{\tilde{y}_{2}^{1}, \ldots, \tilde{y}_{2}^{\tilde{k}_{2}}\right\}=\left\{y_{2}^{k}: y_{2}^{k} \leqslant z_{0}, k=1, \ldots, \bar{q}_{2}^{0}\right\}$ with $\tilde{y}_{2}^{1}<\cdots<\tilde{y}_{2}^{\tilde{k}_{2}}$,

$\left\{\hat{y}_{2}^{1}, \ldots, \hat{y}_{2}^{\hat{k}_{2}}\right\}=\left\{y_{2}^{k}: y_{2}^{k}>z_{0}, k=1, \ldots, \bar{q}_{2}^{0}\right\}$ with $\hat{y}_{2}^{1}<\cdots<\hat{y}_{2}^{\hat{k}_{2}}$.
Then, by the definition of $\bar{q}_{2}^{0}$,

$\tilde{k}_{1} \leqslant \tilde{k}_{2}-1$ and $\hat{k}_{1} \leqslant \hat{k}_{2}-1$,

and

$$
\begin{aligned}
q_{1}^{*} \cdot & \min \left\{G_{1}\left(r_{1}^{*}\right), G_{1}\left(r_{1}^{*}+q_{1}^{*}+1\right)\right\}-\sum_{k=1}^{q_{1}^{*}} G_{1}\left(y_{1}^{k}\right) \\
\leqslant & {\left[\tilde{k}_{1} \cdot G_{1}\left(r_{1}^{*}\right)-\sum_{k=1}^{\tilde{k}_{1}} G_{1}\left(\tilde{y}_{1}^{k}\right)\right] } \\
& +\left[\hat{k}_{1} \cdot G_{1}\left(r_{1}^{*}+q_{1}^{*}+1\right)-\sum_{k=1}^{\hat{k}_{1}} G_{1}\left(\hat{y}_{1}^{k}\right)\right] .
\end{aligned}
$$

Furthermore, if the first equality of (52) holds (i.e., $\left.y_{2}^{\bar{q}_{2}^{0}}=r_{1}^{*}\right)$, then

$$
\begin{aligned}
& \left(\bar{q}_{2}^{0}-1\right) \cdot G_{2}\left(r_{1}^{*}\right)-\sum_{k=1}^{\bar{q}_{2}^{0}-1} G_{2}\left(y_{2}^{k}\right) \\
& =\left[\left(\tilde{k}_{2}-1\right) \cdot G_{2}\left(r_{1}^{*}\right)-\sum_{k=2}^{\tilde{k}_{2}} G_{2}\left(\tilde{y}_{2}^{k}\right)\right] \\
& \quad+\left[\hat{k}_{2} \cdot G_{2}\left(r_{1}^{*}\right)-\sum_{k=1}^{\hat{k}_{2}} G_{1}\left(\hat{y}_{2}^{k}\right)\right] \\
& \geqslant\left[\left(\tilde{k}_{2}-1\right) \cdot G_{2}\left(r_{1}^{*}\right)-\sum_{k=2}^{\tilde{k}_{2}} G_{2}\left(\tilde{y}_{2}^{k}\right)\right] \\
& \quad+\left[\hat{k}_{2} \cdot G_{2}\left(r_{1}^{*}+q_{1}^{*}+1\right)-\sum_{k=1}^{\hat{k}_{2}} G_{1}\left(\hat{y}_{2}^{k}\right)\right],
\end{aligned}
$$

and if the second equality of (52) holds (i.e., $y_{2}^{\bar{q}_{2}^{0}}=$ $\left.r_{1}^{*}+q_{1}^{*}+1\right)$, then

$$
\begin{aligned}
& \left(\bar{q}_{2}^{0}-1\right) \cdot G_{2}\left(r_{1}^{*}+q_{1}^{*}+1\right)-\sum_{k=1}^{\bar{q}_{2}^{0}-1} G_{2}\left(y_{2}^{k}\right) \\
& \geqslant\left[\tilde{k}_{2} \cdot G_{2}\left(r_{1}^{*}\right)-\sum_{k=1}^{\tilde{k}_{2}} G_{2}\left(\tilde{y}_{2}^{k}\right)\right] \\
& \quad+\left[\left(\hat{k}_{2}-1\right) \cdot G_{2}\left(r_{1}^{*}+q_{1}^{*}+1\right)-\sum_{k=1}^{\hat{k}_{2}-1} G_{1}\left(\hat{y}_{2}^{k}\right)\right] .
\end{aligned}
$$

Again using (42)-(43), and (57), if the first equality of (52) holds, then we have

$$
\begin{aligned}
& \left(\tilde{k}_{2}-1\right) \cdot G_{2}\left(r_{1}^{*}\right)-\sum_{k=2}^{\tilde{k}_{2}} G_{2}\left(\tilde{y}_{2}^{k}\right) \geqslant \tilde{k}_{1} \cdot G_{1}\left(r_{1}^{*}\right)-\sum_{k=1}^{\tilde{k}_{1}} G_{1}\left(\tilde{y}_{1}^{k}\right), \\
& \hat{k}_{2} \cdot G_{2}\left(r_{1}^{*}+q_{1}^{*}+1\right)-\sum_{k=1}^{\hat{k}_{2}} G_{2}\left(\hat{y}_{2}^{k}\right) \\
& \geqslant \hat{k}_{1} \cdot G_{1}\left(r_{1}^{*}+q_{1}^{*}+1\right)-\sum_{k=1}^{\hat{k}_{1}} G_{1}\left(\hat{y}_{1}^{k}\right),
\end{aligned}
$$


which contradicts (54) and (55), in view of (58) and (59). Similarly, if the second equality of (52) holds, then

$$
\begin{aligned}
& \tilde{k}_{2} \cdot G_{2}\left(r_{1}^{*}\right)-\sum_{k=1}^{\tilde{k}_{2}} G_{2}\left(\tilde{y}_{2}^{k}\right) \geqslant \tilde{k}_{1} \cdot G_{1}\left(r_{1}^{*}\right)-\sum_{k=1}^{\tilde{k}_{1}} G_{1}\left(\tilde{y}_{1}^{k}\right), \\
& \left(\hat{k}_{2}-1\right) \cdot G_{2}\left(r_{1}^{*}+q_{1}^{*}+1\right)-\sum_{k=1}^{\hat{k}_{2}-1} G_{1}\left(\hat{y}_{2}^{k}\right) \\
& \geqslant \hat{k}_{1} \cdot G_{1}\left(r_{1}^{*}+q_{1}^{*}+1\right)-\sum_{k=1}^{\hat{k}_{1}} G_{1}\left(\hat{y}_{1}^{k}\right),
\end{aligned}
$$

which contradicts (54) and (56), in view of (58) and (60). Therefore, Case 3 is proved.

Proof of Theorem 9. For a simpler exposition, in the following, $r^{*}(\sigma)$ and $q^{*}(\sigma)$ are simply written as $r$ and $q$.

From the proof of Theorem 8 , we have

$$
\begin{aligned}
\frac{\partial r}{\partial \sigma} & =\frac{1}{|A|}\left|\begin{array}{cc}
(r+q) G^{\prime}(r+q) & -b_{1} \\
G^{\prime}(r+q) & -b_{2}
\end{array}\right| \\
& =\frac{-G^{\prime}(r+q)(p+h)}{|A|}\left[-q \phi\left(z_{r}\right)+\int_{r}^{r+q} \phi\left(z_{y}\right) \mathrm{d} y\right]
\end{aligned}
$$

and

$$
\begin{aligned}
\frac{\partial q}{\partial \sigma}= & \frac{1}{|A|}\left|\begin{array}{cc}
-b_{1} & (r+q) G^{\prime}(r+q)-r G^{\prime}(r) \\
-b_{2} & G^{\prime}(r+q)-G^{\prime}(r)
\end{array}\right| \\
= & \frac{1}{|A|}\left[-(p+h)\left(\int_{r}^{r+q} \phi\left(z_{y}\right) \mathrm{d} y-q \phi\left(z_{r+q}\right)\right) \times G^{\prime}(r)\right. \\
& \left.+(p+h)\left(\int_{r}^{r+q} \phi\left(z_{y}\right) \mathrm{d} y-q \phi\left(z_{r}\right)\right) \times G^{\prime}(r+q)\right] .
\end{aligned}
$$

Thus,

$$
\frac{\partial(r+q)}{\partial \sigma}=\frac{-(p+h) G^{\prime}(r)}{|A|}\left(\int_{r}^{r+q} \phi\left(z_{y}\right) \mathrm{d} y-q \phi\left(z_{r+q}\right)\right) .
$$

Recall that the optimal base-stock level $y^{*}$ solves $G^{\prime}(y)=0$. Because $p /(p+h) \geqslant 1 / 2$, we have $r+q>y^{*} \geqslant \mu$. Also, from Zheng (1992), $r<y^{*}$ and $G^{\prime}(r)<0$. Therefore, to prove $\partial(r+q) / \partial \sigma>0$, it suffices to prove

$\int_{r}^{r+q} e^{-(y-\mu)^{2} /\left(2 \sigma^{2}\right)} \mathrm{d} y-q e^{-z_{r+q}^{2} / 2}>0$.

Using the unimodality of $e^{-(y-\mu)^{2} /\left(2 \sigma^{2}\right)}$ and $r+q>\mu$, it suffices to prove

$e^{-z_{r}^{2} / 2}>e^{-z_{r+q}^{2} / 2}$

Suppose contrariwise that

$e^{-z_{r}^{2} / 2} \leqslant e^{-z_{r+q}^{2} / 2}$ which, by $r+q>\mu$, implies that $\mu-r \geqslant r+q-\mu$. Noting that, by (32),

$$
\begin{aligned}
& \int_{2 \mu-(r+q)}^{2 \mu-y^{*}} G^{\prime}(y) \mathrm{d} y \\
& =\int_{2 \mu-(r+q)}^{2 \mu-y^{*}}\left(\frac{p+h}{\sqrt{2 \pi}} \int_{-\infty}^{\left(y^{*}-\mu\right) / \sigma} e^{-s^{2} / 2} \mathrm{~d} s\right. \\
& \left.\quad+\frac{p+h}{\sqrt{2 \pi}} \int_{\left(y^{*}-\mu\right) / \sigma}^{(y-\mu) / \sigma} e^{-s^{2} / 2} \mathrm{~d} s-p\right) \mathrm{d} y \\
& =\int_{2 \mu-(r+q)}^{2 \mu-y^{*}}\left(\frac{p+h}{\sqrt{2 \pi}} \int_{\left(y^{*}-\mu\right) / \sigma}^{(y-\mu) / \sigma} e^{-s^{2} / 2} \mathrm{~d} s\right) \mathrm{d} y \\
& =\int_{2 \mu-(r+q)}^{2 \mu-y^{*}}\left(\frac{p+h}{\sqrt{2 \pi} \sigma} \int_{y^{*}}^{y} e^{-(s-\mu)^{2} /\left(2 \sigma^{2}\right)} \mathrm{d} s\right) \mathrm{d} y \\
& =-\int_{r+q}^{y^{*}}\left(\frac{p+h}{\sqrt{2 \pi} \sigma} \int_{y^{*}}^{2 \mu-y} e^{-(s-\mu)^{2} /\left(2 \sigma^{2}\right)} \mathrm{d} s\right) \mathrm{d} y \\
& =\int_{r+q}^{y^{*}}\left(\frac{p+h}{\sqrt{2 \pi} \sigma} \int_{2 \mu-y^{*}}^{y} e^{-(s-\mu)^{2} /\left(2 \sigma^{2}\right)} \mathrm{d} s\right) \mathrm{d} y \\
& =-\int_{y^{*}}^{r+q}\left[\frac{p+h}{\sqrt{2 \pi} \sigma} \int_{-\infty}^{2 \mu-y^{*}} e^{-(s-\mu)^{2} /\left(2 \sigma^{2}\right)} \mathrm{d} s\right. \\
& \left.\quad+\frac{p+h}{\sqrt{2 \pi} \sigma} \int_{2 \mu-y^{*}}^{y} e^{-(s-\mu)^{2} /\left(2 \sigma^{2}\right)} \mathrm{d} s-p\right] \mathrm{d} y \\
& =-\int_{y^{*}}^{r+q} G^{\prime}(y) \mathrm{d} y, \\
& \left.\frac{p+h}{\sqrt{2 \pi} \sigma} \int_{-\infty}^{y} e^{-(s-\mu)^{2} /\left(2 \sigma^{2}\right)} \mathrm{d} s-p\right] \mathrm{d} y \\
& =
\end{aligned}
$$

we have

$$
\begin{aligned}
G(r+q)-G(r-3|r|) \\
=\int_{r-3|r|}^{r+q} G^{\prime}(y) \mathrm{d} y \\
=\int_{r-3|r|}^{r} G^{\prime}(y) \mathrm{d} y+\int_{r}^{2 \mu-(r+q)} G^{\prime}(y) \mathrm{d} y+\int_{2 \mu-(r+q)}^{2 \mu-y^{*}} G^{\prime}(y) \mathrm{d} y \\
\quad+\int_{2 \mu-y^{*}}^{y^{*}} G^{\prime}(y) \mathrm{d} y+\int_{y^{*}}^{r+q} G^{\prime}(y) \mathrm{d} y \\
=\int_{r-3|r|}^{r} G^{\prime}(y) \mathrm{d} y+\int_{r}^{2 \mu-(r+q)} G^{\prime}(y) \mathrm{d} y+\int_{2 \mu-y^{*}}^{y^{*}} G^{\prime}(y) \mathrm{d} y \\
<\int_{r-3|r|}^{r} G^{\prime}(y) \mathrm{d} y,
\end{aligned}
$$

where $G^{\prime}(y)<0$ for $y<y^{*}$ and $\mu-r \geqslant r+q-\mu$ are used in the last inequality. This contradicts $G(r)=G(r+q)$. Therefore, we have the monotonicity of $r+q$.

\section{Acknowledgments}

The authors thank Jian-Kui Yang and Lei Yang for their help with the numerical examples. They also thank Kevin Shang and Paul Zipkin, as well as the anonymous 
associate editor and referees for their helpful comments and suggestions. This research was supported in part by the National Natural Science Foundation of China under awards 70328001 and 70731003 .

\section{References}

Bagchi, U., J. Hayya, C. Chu. 1986. The effect of lead time variability: The case of independent demand. J. Oper. Management 6 159-177.

Brumelle, L., R. Vickson. 1975. A unified approach to stochastic dominance. W. Ziemba, R. Vickson, eds. Stochastic Optimization in Finance. Academic Press, New York.

Chopra, S., G. Reinhardt, M. Dada. 2004. The effect of lead time uncertainty on safety stocks. Decision Sci. 35 1-24.

Federgruen, A., Y. S. Zheng. 1992. An efficient algorithm for computing an optimal $(r, q)$ policy in continuous review stochastic inventory systems. Oper. Res. 40 808-813.

Gallego, G. 1998. New bounds and heuristics for $(q, r)$ policies. Management Sci. 44 219-233.

Gerchak, Y., D. Mossman. 1992. On the effect of demand randomness on inventories and costs. Oper. Res. 40 804-807.

Gupta, D., W. Cooper. 2005. Stochastic comparisons in production yield management. Oper. Res. 53 377-384.

Iyer, A., A. Jain. 2003. The logistics impact of a mixture of order-streams in a manufacturer-retailer system. Management Sci. 49 890-906.

Jemaï, Z., F. Karaesmen. 2005. The influence of demand variability on the performance of a make-to-stock queue. Eur. J. Oper. Res. 164 195-205.

Karlin, S., A. Novikoff. 1963. Generalized convex inequalities. Pacific J. Math. 13 1251-1279.
Lu, Y., J. S. Song, D. Yao. 2003. Order fill rate, lead time variability, and advance demand information in an assemble-to-order system. Oper. Res. 51 292-308.

Müller, A., D. Stoyan. 2002. Comparison Methods for Stochastic Models and Risks. John Wiley \& Sons, New York.

Ridder, A., E. van der Laan, M. Solomon. 1998. How larger demand variability may lead to lower costs in the newsvendor problem. Oper Res. 46 934-936.

Shaked, M., G. Shanthikumar. 1994. Stochastic Orders and Their Applications. Academic Press, London.

Song, J. S. 1994a. The effect of lead time uncertainty in a simple stochastic inventory model. Management Sci. 40 603-612.

Song, J. S. 1994b. Understanding the lead time effects in stochastic inventory systems with discounted costs. Oper. Res. Lett. 15 85-93.

Song, J. S., D. Yao. 2002. Performance analysis and optimization in assemble-to-order systems with random lead times. Oper. Res. 50 889-903.

Song, J. S., P. Zipkin. 1996. The joint effect of lead time variance and lot size in a parallel processing environment. Management Sci. 42 1352-1363.

Tyworth, J., L. O'Neill. 1997. Robustness of the normal approximation of lead-time demand in a distribution setting. Naval Res. Logist. 44 165-186.

Veinott, A. 1965. The optimal inventory policy for batch ordering. Oper Res. 13 424-432.

Zheng, Y. S. 1992. On properties of stochastic inventory systems. Management Sci. 38 87-102.

Zipkin, P. 1986. Stochastic lead times in continuous time inventory models. Naval Res. Logist. 33 763-774.

Zipkin, P. 2000. Foundations of Inventory Management. McGraw HillIrwin, New York. 